\title{
1 A novel method for calculating ambient \\ 2 aerosol liquid water contents based on \\ 3 measurements of a humidified \\ 4 nephelometer system
}

5 Ye Kuang ${ }^{1,2}$, ChunSheng Zhao ${ }^{1}$, Gang Zhao ${ }^{1}$, JiangChuan Tao ${ }^{1,2}$, Nan $\mathrm{Ma}^{2}$,

$6 \quad$ YuXuan Bian ${ }^{3}$

$7 \quad[1]\{$ Department of Atmospheric and Oceanic Sciences, School of Physics, Peking

8 University, Beijing, China\}

$9[2]\{$ Institute for Environmental and Climate Research, Jinan University, Guangzhou

10511443 , China

[3]\{State Key Laboratory of Severe Weather, Chinese Academy of Meteorological

Sciences $\}$ 


\subsection{Field campaigns}

Datasets from five field campaigns are used in this paper. These campaigns are conducted at three locations on the North China Plain (NCP) during different time periods. The three locations are Wangdu (WD) and Xianghe (XH) in Heibei province and Wuqing (WQ) in Tianjin, and shown in Fig.S1. These three sites are background sites of the NCP. Time periods and locations of these filed campaigns are listed in Table S1.

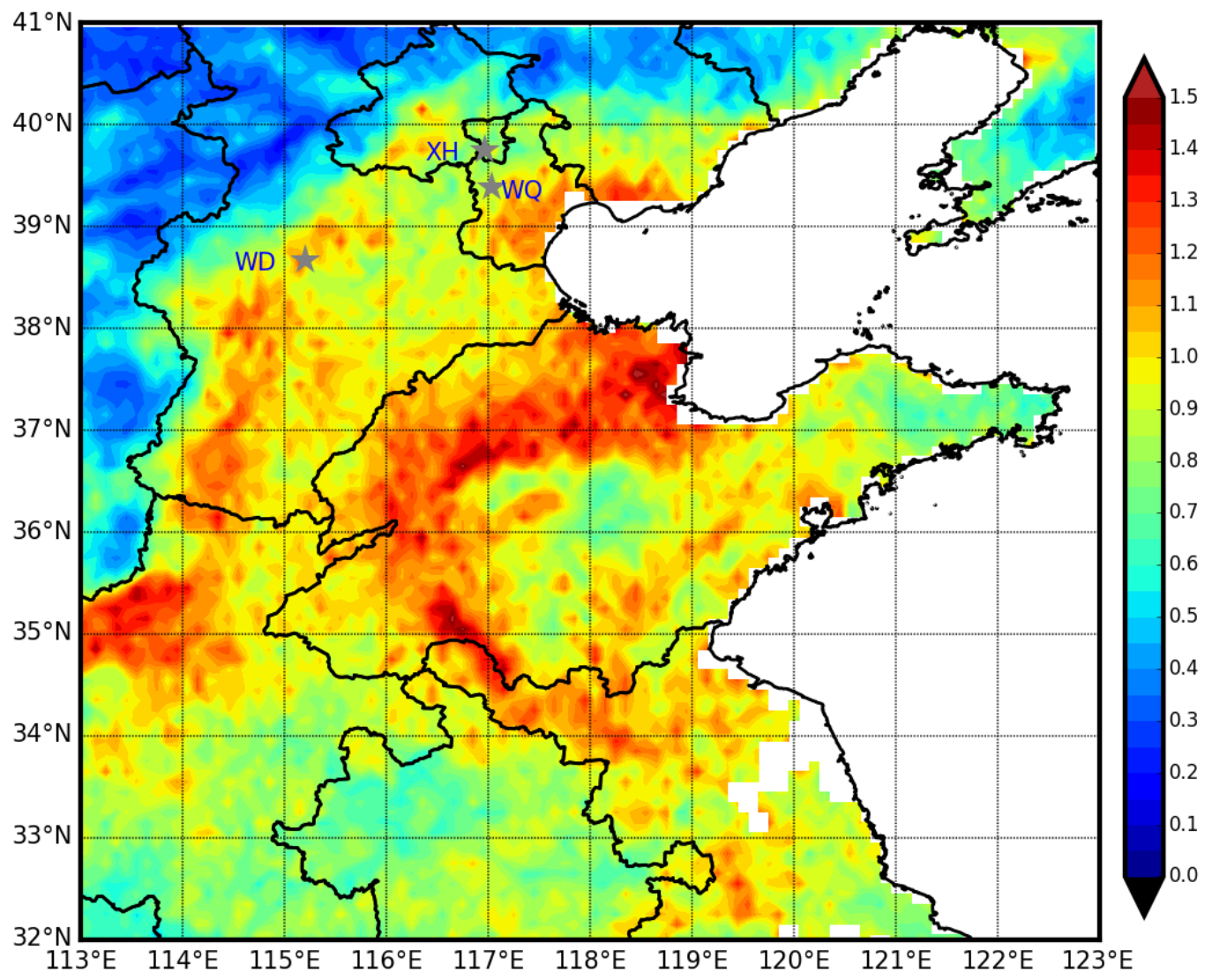

Figure S1. Locations of sites are marked with star markers. Colors represent average distribution of aerosol optical depth at $550 \mathrm{~nm}$ during summer from 2012 to 2014. The dataset of aerosol optical depth at $550 \mathrm{~nm}$ is from Moderate Resolution Imaging Spectroradiometer onboard satellite Aqua. 
Table S1. Locations and time periods of fived field campaigns

\begin{tabular}{|c|c|c|}
\hline Campaign name & Location & Time periods \\
\hline F1 & Wuqing & 7 march to 4 April, 2009 \\
\hline F2 & Wuqing & 12 July to 14 August, 2009 \\
\hline F3 & Xianghe & 22 July to 30 August, 2012 \\
\hline F4 & Xianghe & 9 July to 8 August, 2013 \\
\hline F5 & Wangdu & 4 June to 14 July, 2014 \\
\hline
\end{tabular}

31

During campaign F5, a humidified nephelometer system are available for measuring $\sigma_{s p}$ of aerosol particles in dry state and $f(\mathrm{RH})$. This humidified nephelometer system consists of two nephelometers (TSI Inc., Model 3563) and a humidifier. After passing through a dryer which is capable of reducing the $\mathrm{RH}$ of the sample air to lower than $30 \%$, the sample air was pumped into different instruments. The total and back scattering coefficients in the dry state were measured by the first nephelometer (Neph1). After passing through Neph1, the aerosol particles passed through a humidifier which humidified the sample air through a Gore-Tex tube. The water vapor penetrates through the Gore-Tex tube, which is surrounded by a circulating water layer in a stainless steel tube. The temperature cycle of the circulating water layer was specified and controlled by a water bath. Next, the total and back scattering coefficients at certain RH points were measured by the second nephelometer (Neph2). Two combined RH and temperature sensors (Vaisala HMP110; accuracy of $\pm 0.2{ }^{\circ} \mathrm{C}$ and $\pm 1.7 \%$ for $\mathrm{RH}$ ranges from 0 to $90 \%$, respectively, and accuracy of $\pm 2.5 \%$ for $\mathrm{RH}$ ranges from $90 \%$ to $100 \%$ according to the manufacturer) are placed at the inlet and outlet of Neph2, and the measured RHs and temperatures are defined as $R H_{1} / T_{1}$ and $R H_{2} / T_{2}$, respectively. The dew points at the inlet and outlet of Neph2 are calculated using the measured $R H_{1} / T_{1}$ and $R H_{2} / T_{2}$, and the average value is considered the dew point of the sample air. The sample RH can then be calculated through the derived dew point, and the sample temperature is measured by the sensor inside the sample cavity of the nephelometer. Measurements from the humidified nephelometer system were only available from 21 June, 2014, to 1 July , 2014. 